\title{
Simulation of Extended Source Localization using sLORETA Method for Magnetocardiography
}

\author{
W. Sun and K. Kobayashi \\ Faculty of Engineering, Iwate University, 4-3-5 Ueda, Morioka, Iwate 020-8551, Japan
}

\begin{abstract}
In this study, cardiac source localization was simulated using the spatial filter method. Three types of spatial filters were obtained using the standardized low-resolution brain electromagnetic tomography (sLORETA) method, based on different examination procedures. In Type A filter, the examination was conducted at the front of the torso. In both Type B and Type C filters, the examinations were conducted at the front and back of the torso; however, the distance from the frontal observation plane to the center of the heart model was different for each type. In the simulation experiments, first the goodness of fit (GOF) value was introduced to determine the proper threshold for each spatial filter. Then, single and multiple dipole sources were simulated at different depths with and without noise. The extension of the solutions computed using these spatial filters was investigated. Finally, the performances of these spatial filters, with the corresponding averaged thresholds, were evaluated using the GOF. Type B and Type C spatial filters demonstrated reduction in the extension of source dependency on source depth and improvement in the accuracy of source localization with noisy data.
\end{abstract}

Key words: Magnetocardiography, sLORETA method, source localization, spatial filter

\section{Introduction}

Magnetocardiography (MCG) is a noninvasive technique that detects the magnetic field generated by the electrical activity in the heart ${ }^{1}$. Recently, MCG has been attracting a lot of attention in relation to the early detection of heart diseases. As MCG is a multichannel measurement technique and as the signals are not affected by the shape of the lungs and torso ${ }^{2)}$, it has high potential for clinical applications. MCG aims at obtaining functional mapping, starting from the visualization of signal sources. Because the MCG inverse problem is ill posed and does not have a unique solution, the estimation of MCG signal sources is the biggest challenge for its application ${ }^{3)}$. MCG signals are generated by currents flowing within the myocardial fibers during cardiac activity ${ }^{4)}$. Therefore, we can consider that an extended source model will be suitable for estimating MCG sources.

The spatial filter methods ${ }^{5,6)}$ were developed for MCG source localization, and could be used to obtain a reliable three-dimensional (3D) outline of the heart. Among the many spatial filter methods, standardized low-resolution brain electromagnetic tomography (sLORETA $)^{7)}$ is widely used for visualizing brain activity, as it has zero localization error for a single dipole with noiseless data. However, it has some limitations, especially under noisy conditions and in case of deep sources $^{8,9)}$. For conventional method, MCG examination is just conducted in the front of the torso, in order to obtain strong signals. In a previous study ${ }^{10}$, the spatial filter always favors the sources that are close to the magnetic field sensors and leads to reconstruction of a large extension for deep sources. Namely, the extension of the source depends on the source depth. MCG can detect heart signals not only at the front but also at the back of the torso, even though the signals detected at the back of the torso are very weak ${ }^{11)}$. The assessments of the spatial filter based on the different examination method have not been studied. In order to assess the source localization abilities of the spatial filters, and investigate the dependency of source extension on the source depth, the simulation study is needed.

In this study, we make sure the distances from the frontal and back observation planes to the heart are the same, to reduce the dependency of the source extension on the source depth, and obtain a spatial filter that can estimate a cardiac source well with noisy data.

\section{Simulation Model}

In this study, we simulated MCG source localization using the sLORETA method based on a conventional 64-channel MCG system. Superconducting quantum interference device (SQUID) modules were aligned in an $8 \times 8$ configuration to create a square observation area sized $175 \mathrm{~mm} \times 175 \mathrm{~mm}^{12)}$.

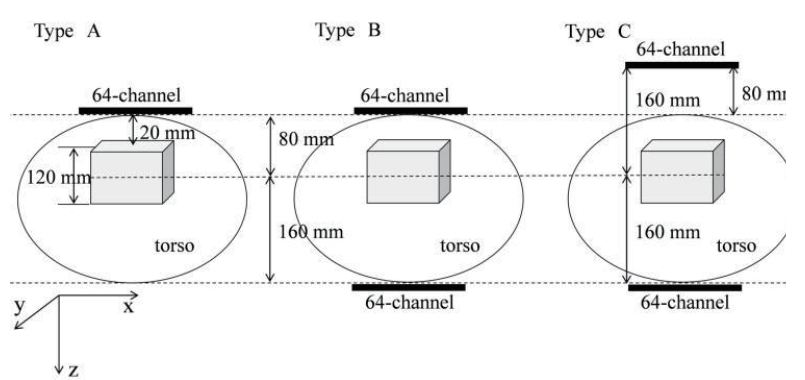

Fig. 1 Frontal and back observation planes and the position of heart in torso. 
Simulations were carried out for the 64-channel MCG system within a $168 \mathrm{~mm} \times 168 \mathrm{~mm} \times 120 \mathrm{~mm}$ volume. Six thousand six hundred and fifteen voxels were divided with the size of each voxel being $8 \mathrm{~mm} \times 8$ $\mathrm{mm} \times 8 \mathrm{~mm}$. Fig. 1 shows three types examination procedures. Type A is the conventional examination method, which is only conducted at the front of the torso. In both Type B and Type C, the examinations are conducted not only at the front but also at the back of the torso; however, the distance from the frontal observation plane to the center of the heart model is different for each type. For Type C, in order to compensate for the varying sensitivities of the sensors to the current sources at different depths, we make sure the distances from the frontal and back observation planes to the heart are the same. The distance from the frontal observation plane to the center of the heart model was set as $80 \mathrm{~mm}$ for Type A and Type B and as $160 \mathrm{~mm}$ for Type C. The distance from the back observation plane to the center of the heart model was set as $160 \mathrm{~mm}$ for Type B and Type C. For the forward problem, we calculated the lead-field matrix $\mathbf{K}$ using the Biot-Savart Law.

\section{Methods}

sLORETA is a spatial filter method used to solve the ill posed problem. It can obtain a blurred source image by using a linear inverse operator ${ }^{7)}$.

$$
\boldsymbol{\Phi}=\boldsymbol{K} \boldsymbol{J}+\boldsymbol{n}
$$

where $\Phi$ is the detected signals, $\mathbf{K}$ is the lead-field matrix, $\mathbf{J}$ is the original current sources within the heart, and $\mathbf{n}$ is the noise at each sensor. Using the well-known minimum norm estimate (MNE) method, the estimated current sources $\hat{\jmath}$ can be written as:

$$
\hat{\boldsymbol{J}}=\boldsymbol{G} \boldsymbol{\Phi}=\boldsymbol{K}^{\mathrm{T}}\left(\boldsymbol{K} \boldsymbol{K}^{\mathrm{T}}+\lambda^{2} \boldsymbol{I}\right) \boldsymbol{\Phi}
$$

where $\mathbf{G}$ is a spatial filter matrix, $\mathrm{T}$ denotes the matrix transpose, $\lambda^{2}$ is the Tikhonov regularization parameter, and I represents an identity matrix. The sLORETA solution can be obtained from the standardization of the

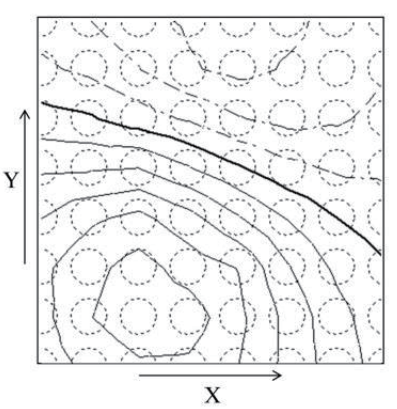

(a)

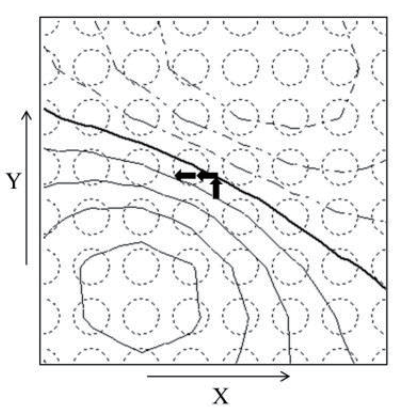

(b)
Fig. 2 (a) Isomagnetic field map of MCG wave at R peak. (b) Isomagnetic field map of multiple dipole sources. GOF of two patterns is 0.93 . One step of the isomagnetic field contour line is $10 \mathrm{pT}$.
MNE solution using its variance in the following way:

$$
\hat{\boldsymbol{J}}_{\text {SLORETA,i }}=\boldsymbol{S}_{\mathrm{ii}}{ }^{-1 / 2} \hat{\boldsymbol{J}}_{\mathrm{i}}
$$

where $\mathbf{S}_{\mathrm{ii}}$ is the diagonal element of the covariance matrix $\mathbf{S}$ with

$$
\boldsymbol{S}=\boldsymbol{G} \boldsymbol{K}
$$

In this study, we first calculate the lead-field matrix $\mathbf{K}$ that describes the sensitivity of the sensors to each current source location. For each current source location, $\mathrm{K}$ includes two columns that represent the $\mathrm{X}$ component and Y component, respectively. Then, Type A, Type B, and Type $\mathrm{C}$ spatial filters are obtained using the sLORETA method.

In the coordinate system, the center point of the top voxels layer is $(0,0,20)$ and the unit is $\mathrm{mm}$. A single dipole source $(0,0,20)$ in the $\mathrm{X}$ direction is simulated by changing the depth in $8 \mathrm{~mm}$ increments.

Since the real cardiac sources are considered to be extended sources, simulations with multiple dipoles are also performed. A normal MCG wave is obtained using the Iwate create project 64 -channel MCG system ${ }^{11)}$. An isomagnetic field map of the MCG wave at the R peak is shown in Fig. 2(a). The generated magnetic field of the multiple sources is shown in Fig. 2(b). Multiple sources- $(-16,8,20)$ in the $\mathrm{X}$ direction, $(-8,8,20)$ in the $\mathrm{X}$ direction, and $(0,0,20)$ in the $\mathrm{Y}$ direction-are simulated by changing the depth in $8 \mathrm{~mm}$ increments.

\section{Simulation Results and Discussions}

\subsection{Simulation without Noise}

Fig. 3 shows the simulation results of a single dipole at different depths (depth $=28 \mathrm{~mm}, 76 \mathrm{~mm}$, and 124 $\mathrm{mm}$ ). The relative strength was normalized by the maximum of the solutions. The white rectangle denotes the maximum of the solutions. All the maximum

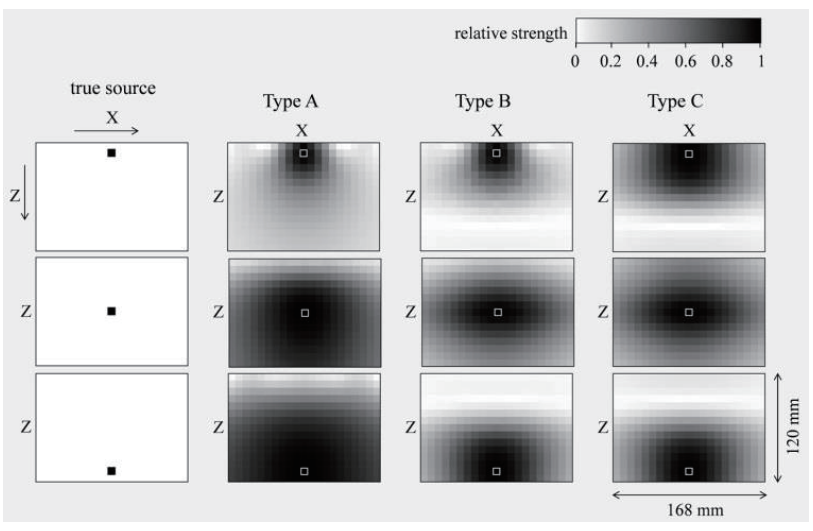

Fig. 3 Left column is the true source in the $\mathrm{X}$ direction placed at different depths (from top to bottom depth $=28$ $\mathrm{mm}, 76 \mathrm{~mm}$, and $124 \mathrm{~mm}$ ). The three right columns are cross sections of simulation results using Type A, Type $\mathrm{B}$, and Type $\mathrm{C}$ spatial filters. The white rectangle denotes the maximum of the solutions. 
solutions are located at real source positions. It means that there is no localization error without noise. However, the solutions have large spatial extensions. Obviously, the extensions of the solutions computed by the Type A spatial filter have larger dependencies on the depths of the true source than that computed by the Type B and Type C spatial filters. Because a source that is far from the sensor contributes less to the measured data than one that is near the sensor, the solution needs large extensions to fit the measured data. By contrast, Type B and Type C spatial filters produce solutions with extensions that have fewer dependencies on the depths of true sources.

For an extended source, a threshold of relative strength has to be determined. The full width at half maximum (FWHM) is used as the threshold ${ }^{13,14)}$. It means that only the excess $50 \%$ of the maximum sources are analyzed to avoid influence from the weak insignificant source estimates. In our study, the goodness of fit (GOF) value is introduced to determine the proper threshold. GOF describes the similarities in the measured and estimated signals at all $\mathrm{N}$ sensors ${ }^{15)}$. GOF is defined as follows:

$$
G O F=1-\sum_{i=1}^{N}\left(\boldsymbol{B}_{\text {meai }}-\boldsymbol{B}_{\text {cal,i }}\right)^{2} / \sum_{i=1}^{N} \boldsymbol{B}_{\text {cal,i }}^{2}
$$

where $\boldsymbol{B}_{\text {mea,i }}$ denotes the measured magnetic field and

Table 1 Threshold of relative strength simulated with the true source at different depths when the corresponding GOF is close to 1. (All of the GOF is 0.99 in this study).

\begin{tabular}{cccc}
\hline \hline depth $(\mathrm{mm})$ & Type A & Type B & Type C \\
\hline 20 & 0.847 & 0.892 & 0.940 \\
28 & 0.830 & 0.856 & 0.946 \\
36 & 0.850 & 0.860 & 0.950 \\
44 & 0.866 & 0.870 & 0.940 \\
52 & 0.902 & 0.880 & 0.925 \\
60 & 0.910 & 0.883 & 0.917 \\
68 & 0.924 & 0.885 & 0.911 \\
76 & 0.934 & 0.886 & 0.900 \\
84 & 0.936 & 0.910 & 0.910 \\
92 & 0.942 & 0.925 & 0.920 \\
100 & 0.942 & 0.929 & 0.931 \\
108 & 0.938 & 0.938 & 0.940 \\
116 & 0.937 & 0.950 & 0.952 \\
124 & 0.927 & 0.950 & 0.951 \\
132 & 0.923 & 0.947 & 0.947 \\
average & 0.91 & 0.90 & 0.93 \\
\hline \hline
\end{tabular}

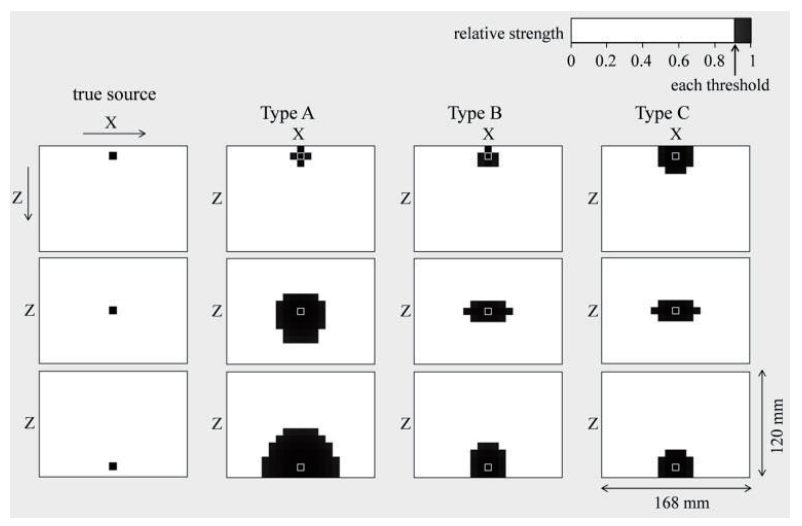

Fig. 4 Left column is the true source in the $\mathrm{X}$ direction placed at different depths (from top to bottom depth $=28 \mathrm{~mm}, 76 \mathrm{~mm}$, and $124 \mathrm{~mm}$ ). The three right columns are cross sections of simulation results using Type A, Type B, and Type C spatial filters (threshold $=0.91,0.90$, and 0.93, respectively). The white rectangle denotes the maximum of the solutions.

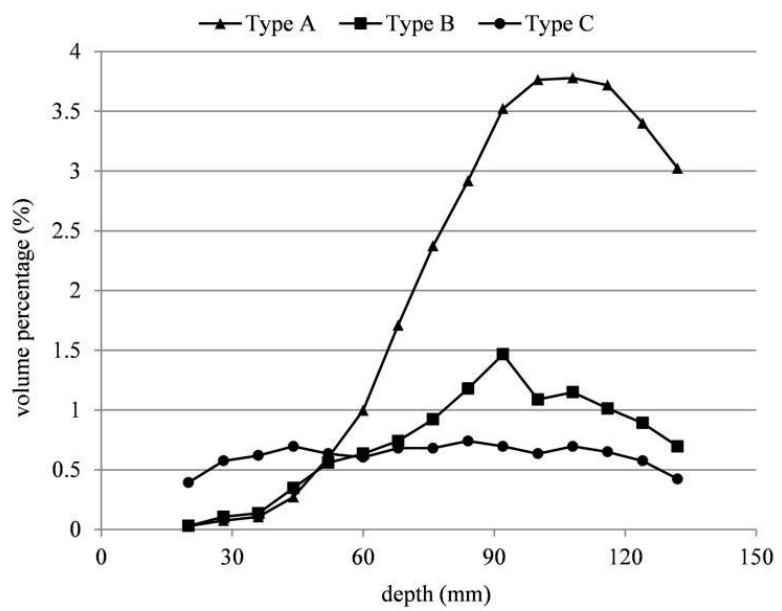

Fig. 5 Relationship of volume percentage and depth of true source.

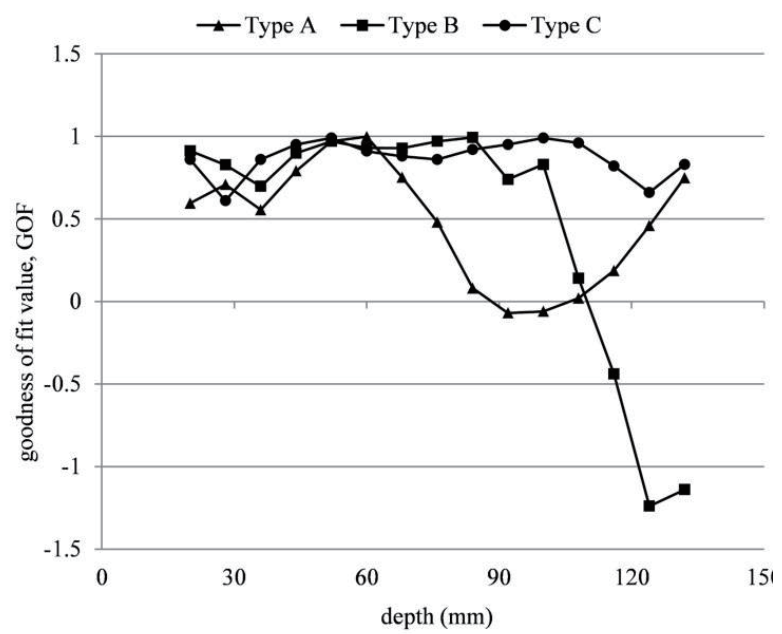

Fig. 6 GOFs of solutions computed using Type A, Type B, and Type $\mathrm{C}$ spatial filters when the true source is placed at different depths. 
$\boldsymbol{B}_{\text {cal,i }}$ denotes the calculated magnetic field at the $i^{\text {th }}$ sensor. A high GOF indicates that the estimated sources can explain the measured data well. The threshold values with true sources at different depths are shown in Table 1, when the corresponding GOF is close to 1 . The average value is chosen for computing the solutions. The relative strength, which does not exceed the average value of thresholds, is considered to be 0 .

In the simulation, a single dipole source in the $\mathrm{X}$ direction is placed at different depths (depth $=28 \mathrm{~mm}$, $76 \mathrm{~mm}$, and $124 \mathrm{~mm}$ ). Fig. 4 shows the cross sections of the solutions computed by Type A, Type B, and Type C spatial filters. The extension of the solution has a large dependency on the depth of the true source for the Type A spatial filter because of the varying solid angles between the dipole at different depths and the observation plane. In addition, the solutions computed by the Type B and Type C spatial filters show smaller extensions in the $\mathrm{Z}$ direction than that computed by the Type A spatial filter, owning to the data measured from

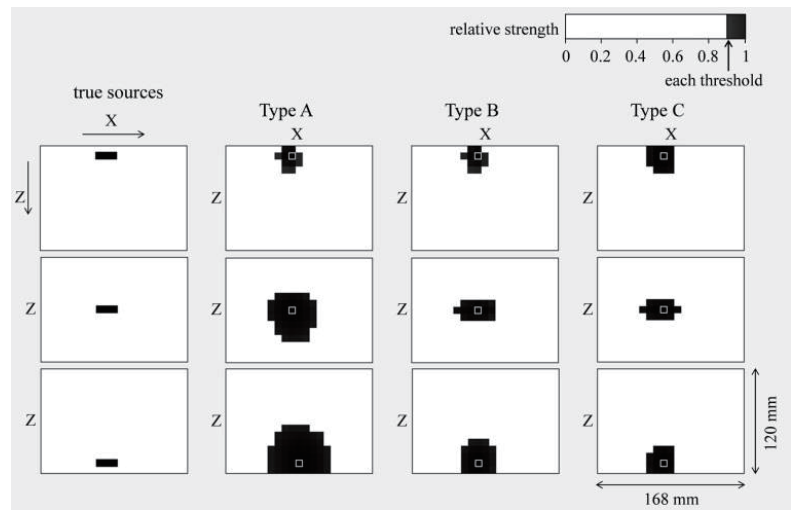

Fig. 7 Left column is the true sources at different depths (from top to bottom depth $=28 \mathrm{~mm}, 76 \mathrm{~mm}$, and $124 \mathrm{~mm}$ ). The three right columns are cross sections of simulation results using Type A, Type B, and Type $\mathrm{C}$ spatial filters (threshold $=0.91,0.90$, and 0.93 , respectively). The white rectangle denotes the maximum of the solutions.

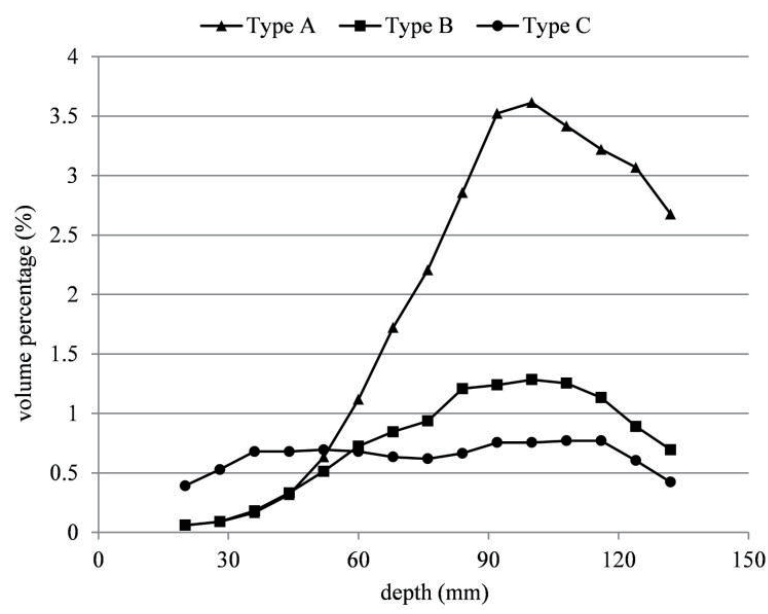

Fig. 8 Relationship of volume percentage and depths of true sources.

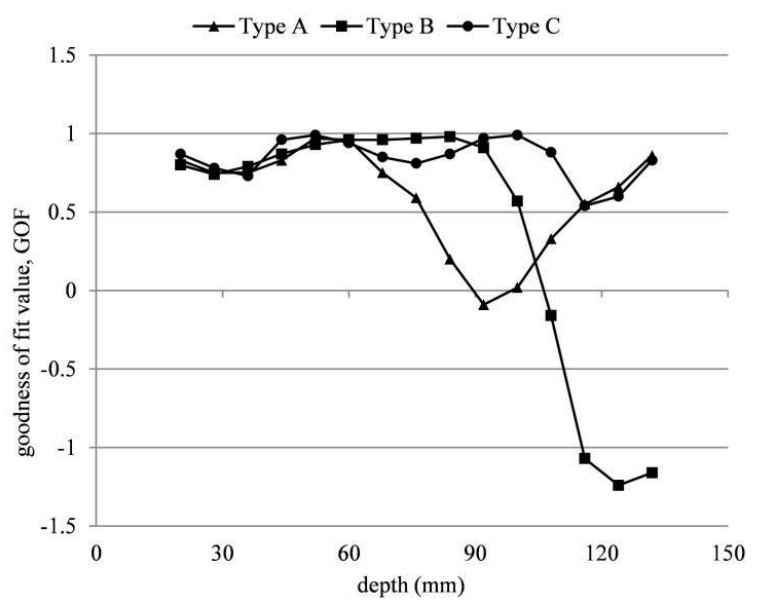

Fig. 9 GOFs of solutions computed using Type A, Type B, and Type C spatial filters when the true sources are placed at different depths.

the back of torso. Because the distances from the frontal and back observation planes to the heart model are the same, the extensions show less dependency on the depth of the true source for the Type C spatial filter.

By computing the volumes of the voxels in which the solutions are localized, the relationship between the volume percentage and the true source depth can be obtained as shown in Fig. 5. The extensions of solutions show less dependency on the depth of the true source for the Type B and Type C spatial filters. The GOFs of Type A, Type B, and Type C spatial filters with the proper thresholds are shown in Fig. 6. The GOF values of the Type A and Type B spatial filters are far away from 1 and even drop to negative values for deep sources. Therefore, the Type C spatial filter shows better performance.

Fig. 7 shows the cross sections of the solutions computed using the Type A, Type B, and Type C spatial filters. By computing the volumes of the voxels in which the solutions are localized, the relationship between the volume percentage and the true source depth can be obtained as shown in Fig. 8. Obviously, the Type B and Type C spatial filters show extensions that have less dependency on the depth of the true sources, compared to the Type A spatial filter. Fig. 9 shows the GOFs of the Type A, Type B, and Type C spatial filters simulated using sources at different depths. The GOFs of Type A and Type B spatial filters are far away from 1 and even drop to negative values for deep sources. It means that these two spatial filters cannot fit the measured data well for deep sources. By contrast, the GOF of the Type $\mathrm{C}$ spatial filter has a good performance despite the slight drop in the case of deep sources.

\subsection{Simulation with Noisy Data}

Since the MCG inverse problem is ill posed, weak noise signals can cause large changes in the solution. In this study, Gaussian white noise is added to the simulated magnetic field data and the inverse solutions 
are computed using the above three spatial filters. The SNR ranges from 0 to 40 in $5 \mathrm{~dB}$ increments. The SNR is defined as:

$$
S N R=10 \log \left\{\operatorname{var}\left(\boldsymbol{B}_{\text {exact }}\right) / \sigma^{2}\right\}
$$

where $\boldsymbol{B}_{\text {exact }}$ is the variance of the simulated noise-free measurements and $\sigma^{2}$ is the variance of the added Gaussian white noise. In this study, for a single dipole source, the localization error is the distance from the maximum estimated source to the true source. For multiple sources, the localization error is the distance from the maximum estimated source to the center point of the true sources. The mean value is obtained from 100 calculations. In order to obtain the same measurement conditions, the same $\sigma$ value of noise is set up for these three spatial filters at a certain noise level. Since the magnitudes of the signals detected at the frontal and back observation planes are different, the SNR is different. In this study, the $\mathrm{x}$-axis values of Fig. 10 and Fig. 11 are calculated only using the measurement data that obtained based on Type A examination procedure.

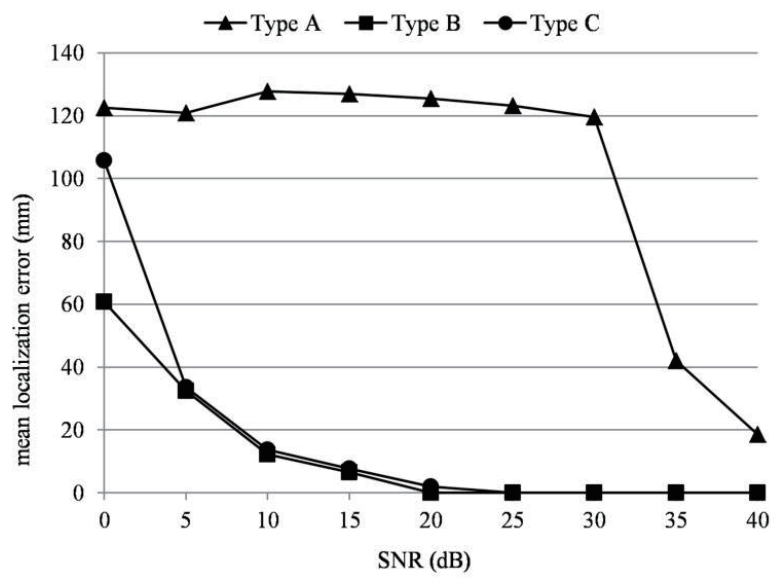

Fig. 10 Mean localization error of single dipole source $($ depth $=124 \mathrm{~mm})$ with different noise levels.

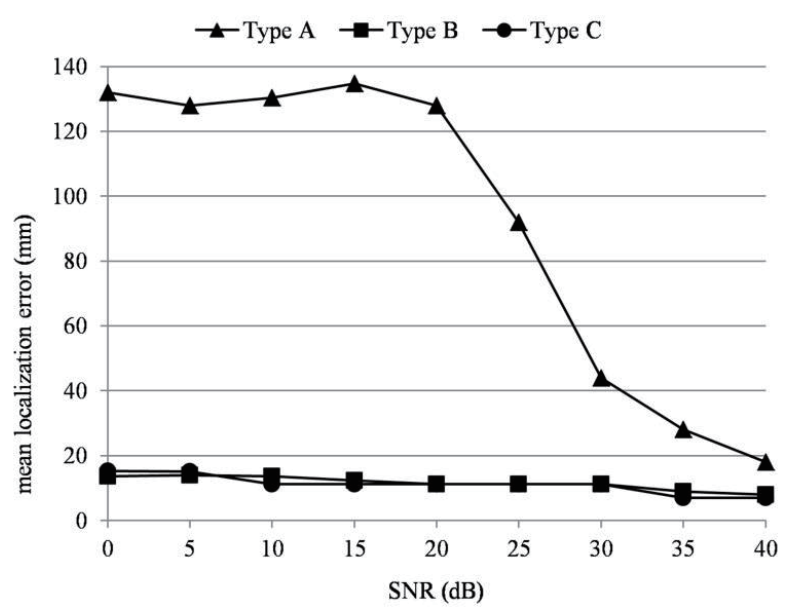

Fig. 11 Mean localization error of multiple sources (depth $=124 \mathrm{~mm})$ with different noise levels.
Fig. 10 and Fig. 11 show the simulation results of a single dipole source and multiple sources, respectively. The single dipole source and the multiple sources are placed at relatively deep positions with a depth of 124 $\mathrm{mm}$. For single dipole source estimation, very weak noise led to Type A spatial filter generating a large localization error. By contrast, the localization errors of Type B and Type C spatial filters were stable and about 0 at $20 \sim 40 \mathrm{~dB}$. For multiple sources estimation, the localization errors of Type B and Type C spatial filters were stable at $0 \sim 40 \mathrm{~dB}$. Apparently, the Type B and Type $\mathrm{C}$ spatial filters have improved source localization accuracies. It may be owing to the data obtained from the back of the torso. In addition, Type B and Type C spatial filters have good performances for multiple source localizations even at high noise levels. Hence, they are fit for estimating multiple sources.

\section{Conclusion}

In this study, Type A, Type B, and Type C spatial filters were obtained using the sLORETA method based on three types of MCG examination procedures. The GOF was introduced to determine the proper threshold for each spatial filter. The extensions of the solutions computed using these spatial filters with the corresponding thresholds were investigated. In the simulation of a single dipole and multiple dipoles placed at different depths, the Type $\mathrm{C}$ spatial filter demonstrated an extension that had less dependency on the depth of the true source. In addition, the GOF of the Type C spatial filter was close to 1 even for deep sources. It meant that the Type $\mathrm{C}$ spatial filter had the best performance for extended source estimation, compared to Type A and Type B spatial filters. From the results of the simulations with noisy data, we found that the Type $\mathrm{B}$ and Type $\mathrm{C}$ spatial filters had improved source localization accuracies and that they were fit for estimating extended sources. The reduction of the source extension for deep sources and the improvement of the source localization accuracies owned to the examination at the back of the torso. The same distance from the frontal and back observation planes led to the less dependency of the source extension on the source depth. In the future, we hope to detect the real MCG signals and estimate the cardiac source using Type B and Type $\mathrm{C}$ spatial filters.

Acknowledgements This work was supported by the Japan Society for the Promotion of Science KAKENHI (Grants-in-Aid for Scientific Research (C)) Grant Number JP26350535.

\section{References}

1) J. S. W. Kwong, B. Leithäuser, J.-W. Park, and C.-M.Yu: Int. J. Cardiol., 167, 1835 ( 2013).

2) K. Nakai et al.: Int. J. Cardiovasc. Imaging, 21, 555 (2005).

3) V. Mäntynen, T. Konttila, and M. Stenroos: Phys. Med. Biol., 59, 7141 (2014). 
3) M. De Melis et al.: J. Magn. Soc. Jpn., 34, 453 (2010).

4) I. Tavarozzi et al.: Ital. Heart J., 3, 75 (2002).

5) K. Nakai et al.: Int. J. Cardiovasc. Imaging, 22, 573 (2006).

6) G. Shetty and M K: Int. J. Emerg. Technol. Innov. Res., 2, 1156 (2015).

7) R. D. Pascual-Marqui: Methods Find. Exp. Clin. Pharmacol., 24,5 (2002).

8) E. Palmero-Soler, K. Dolan, V. Hadamschek and P. A. Tass: Phys. Med. Biol., 52, 1783 (2007).

9) R. Terusawa et al.: J. Magn. Soc. Jpn., 36, 272 (2012).
10) M. Yoshizawa, K. Nakai, K. Kobayashi, Y. Nakamura, Y. Uchikawa: J. Jpn. Biomagn. Bioelectromagn. Soc., 15, 109 (2002).

11) K. Yoshida et al.: J. Arrhythm., 31, 345 (2015).

12) M. Yoshizawa et al.: Phys. C: Supercond. Appl., 426, 1572 (2005).

13) F.-H. Lin et al.: NeuroImage, 31, 160 (2006).

14) M. Fuchs, M. Wagner, T. Kohler, and H.-A. Wischmann: J. Clin. Neurophysiol. 16, 267 (1999).

15) K. Pesola et al.: Med. Biol. Eng. Comput., 37, 2 (1999).

Received Jan. 19, 2017; Accepted May 15, 2017. 